\title{
Enquête psycho-langagière sur les effets de l'humour en publicité
}

A Psychological Discourse Analysis Investigation: Humoristic Strategies and Advertisement

\section{Claude Chabrol et Pierre Vrignaud}

\section{(2) OpenEdition}

Journals

Édition électronique

URL : http://journals.openedition.org/questionsdecommunication/7695

DOI : 10.4000/questionsdecommunication.7695

ISSN : 2259-8901

Éditeur

Presses universitaires de Lorraine

Édition imprimée

Date de publication : 1 décembre 2006

Pagination : 135-156

ISBN : 978-2-86480-828-2

ISSN : 1633-5961

\section{Référence électronique}

Claude Chabrol et Pierre Vrignaud, « Enquête psycho-langagière sur les effets de l'humour en publicité ", Questions de communication [En ligne], 10 | 2006, mis en ligne le 01 décembre 2006, consulté le 22 mars 2021. URL : http://journals.openedition.org/questionsdecommunication/7695 DOI : https://doi.org/10.4000/questionsdecommunication.7695 


\title{
$>$ DOSSIER
}

CLAUDE CHABROL

Groupe de recherche en psychologie de la communication

Université Paris 3

c.chabrol@wanadoo.fr

PIERRE VRIGNAUD

Groupe de recherche en psychologie de la communication

Université Paris 10

vrignaud.pierre@wanadoo.fr

\section{ENQUÊTE PSYCHO-LANGAGIÈRE SUR LES EFFETS DE L'HUMOUR EN PUBLICITÉ}

\begin{abstract}
Résumé. - L'enjeu de cette contribution était de réunir les apports des sciences du langage et de la psychologie sociale de la communication persuasive afin d'évaluer les effets des stratégies humoristiques en réception. Pour ce faire, nous avons conçu un plan d'enquête qui cherchait à mettre en évidence les effets des visées d'effets de connivence - ludique, critique et cynique - , croisées avec une variable opérationnalisant la «familiarité/non-familiarité » liée au contrat de lectorat. Trois groupes indépendants de 25 sujets réagissaient à un ensemble de quatre publicités humoristiques. Deux d'entre elles correspondaient à des publicités françaises largement diffusées, deux autres à des publicités espagnoles, non connues de nos sujets, ce qui permettait de tester l'effet du facteur « familiarité culturelle ». Les limites des résultats quantitatifs (analyse de variance pour un plan à mesures répétées) nous ont conduits à faire une analyse qualitative des données. La variabilité interindividuelle dans les groupes face aux stratégies humoristiques pourrait être liée à l'interaction des variables sémiotiques et psychologiques.
\end{abstract}

Mots clés. - Stratégies humoristiques, effets de connivence, contrat de lectorat, variabilité interindividuelle. 


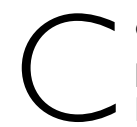

omme le rappelle Patrice Georget (2004: 74), « I'humour en publicité, d'après une enquête réalisée auprès des praticiens de la publicité aux États-Unis, tient une grande place dans leur esprit : $94 \%$ d'entre eux estiment que l'humour permet d'attirer l'attention des consommateurs, $38 \%$ qu'il permet d'accroitre la compréhension du message publicitaire, et $62 \%$ déclarent que les publicités humoristiques sont les plus efficaces sur le plan de la persuasion $\quad$ ». Or, les études psychologiques en réception ne permettent pas d'être aussi optimiste sur les effets positifs de l'humour quant à l'efficacité comportementale attendue. Si l'humour joue clairement sur l'attention, et donc favorise une mémorisation d'éléments divers (pas nécessairement la marque ou le produit !), il n'entraîne pas une meilleure compréhension et, plus profondément, ne produit «pas systématiquement un avantage sur le plan de l'attitude : à l'égard de l'annonce, de la marque et de l'intention d'achat » (ibid.).

Bref, aujourd'hui, il s'agit plutôt pour les psychologues d'examiner quelles sont les conditions sémiotiques et psychologiques qui rendent l'usage de l'humour efficace, au plan des conduites comme des attitudes et des jugements. Les attitudes préalables du destinataire vis-à-vis de la marque ou de la catégorie de produits (Georget, Chabrol, 2000), ou son implication dans la tâche de traitement en fonction de ses enjeux, vont intervenir dans le traitement comme certaines dimensions personnelles cognitives et affectives. Ainsi « le besoin de cognition » (Cacioppo, Petty, 1982) prédispose les personnes en ayant un fort besoin à apprécier davantage les tâches qui exigent un effort cognitif élevé, ou « le self monitoring », cette tendance plus ou moins forte à adapter leur comportement et leurs jugements en fonction des attentes supposées de leur environnement social actuel (Snyder, DeBono, 1985)'. Yong Zhang (1996: 531-545) a montré que l'effet de l'humour dans la publicité changeait selon le niveau de besoin de cognition dans le public. Celui-ci est plus important lorsque les destinataires ont un besoin de cognition faible, car les stratégies humoristiques utilisent peu et mal les arguments «forts $»^{2}$ attendus par ceux qui ont un besoin de cognition élevé. Mark

\footnotetext{
' «Le besoin d'humour » qui semble moduler les attitudes et comportements des individus face à l'humour publicitaire pourrait être lié au self-monitoring, car la tendance à générer et chercher l'humour est socialement renforcée dans les groupes sans activité importante.

${ }^{2}$ En psychologie, la catégorisation argumentative « fort vs faible » repose généralement sur la convergence entre une analyse sémantique fondée sur l'idée que les arguments «forts » sont ceux qui expriment le plus logiquement et rationnellement possible les avantages évidents, techniques et économiques du produit, donc « sémantiquement » apparentés ou/et de plus classés comme tels par des juges de la population concernée.
} 
Snyder et Kenneth G. DeBono (1985) ont mis en valeur la sensibilité de sujets à self monitoring élevé envers les publicités qui manipulaient les univers symboliques associés au produit de façon très vague (non sémantiquement fondée par rapport au produit), comme c'est souvent le cas de l'humour en publicité. On posera (Soulages, 2006) que l'humour décalé et provocateur peut apparaitre comme un moyen de marquage social, de distinction à l'égard du plus grand nombre supposé timoré et conformiste.

A contrario, on a pu prouver les effets positifs de stratégies humoristiques sémantiquement fondées par opposition à celles qui ne le seraient pas. Ainsi Stephen M. Smith (1993) trouve-t-il des effets différenciés sur le type de traitement du message en fonction du type d'humour mobilisé. "L L'humour accessoire », non lié aux contenus sémantiques du message, serait moins favorable que «l'humour apparenté », lié sémantiquement aux arguments du message. Par ailleurs, d'autres recherches démontrent l'impact sur les traitements des co-textes d'exposition dans lesquels se produisent les intrusions publicitaires, et en particulier des co-textes qui produisent une " humeur positive ». Selon les cas, ceux-ci peuvent entraîner des traitements périphériques des messages publicitaires quand les motivations à traiter ou les capacités sont faibles ou centraux, mais biaisés quand elles sont toutes deux fortes et que l'information sur l'objet est ambiguë, ce qui est très fréquent avec les énoncés humoristiques (Georget, 2004 : 76 ; Wegener, Petty, Smoak, Fabrigar, 2004 : 19). Maintenant, si l'humeur positive est supposée provoquée par le message humoristique lui-même, il faudra en savoir plus sur les rapports entre types de messages humoristiques et degré d'humeur positive, sans exclure l'apparition d'une humeur neutre ou négative.

Tous ces éléments rendent nécessaire une meilleure articulation de l'approche psycho-sociale aux apports des sciences du langage. II est difficile de faire une inférence sur l'impact d'une variable psychologique, comme la force de l'attitude initiale ou le degré de pertinence personnelle à partir d'un effet positif sur les traitements, les attitudes finales ou les comportements vis-à-vis d'un produit et/ou d'une marque, sans maîtriser les facteurs sémio-linguistiques qui peuvent interagir avec elle, soit les formes et variétés très diverses d'humour qui sont loin de provoquer les même résultats chez tous les individus. Dans cette contribution, on focalisera d'abord l'attention sur les stratégies humoristiques elles-mêmes, dans la continuité des contributions de Patrick Charaudeau (2006) et Jean-Claude Soulages (2006) dans la présente livraison de Questions de communication, que le lecteur pourra consulter préalablement. Considérons que l'étude qui suit ne peut être que le premier moment d'une investigation beaucoup plus ample. 


\section{Un modèle pragma-sémiotique de la production humoristique}

Patrick Charaudeau (2006) propose un modèle dans sa contribution «Des catégories pour l'humour ? » ${ }^{3}$ qui reprend et développe amplement les premiers résultats des travaux du groupe de travail franco-espagnol « Picasso », et les reconfigure en les confrontant aux traditions rhétoriques et philosophiques sur l'humour. C'est ce modèle qui a servi à nos plus récentes analyses et qui est appliqué dans nos enquêtes en cours, complété par son application au domaine de la publicité par Jean-Claude Soulages (2006). Même si les niveaux sémiotiques et sémantiques (thématiques) y sont représentés, c'est le point de vue pragmatique qui est privilégié puisque la parole humoristique est définie par la visée énonciative d'un « effet de connivence ». Patrick Charaudeau (2006) met au premier plan l'intentionnalité d'une visée de sens partagé. Cette visée générique peut être modulée selon plusieurs types de connivence.

I/ Soit une visée ludique d'un effet de connivence de même type qui, pour Patrick Charaudeau (2006 : 36), « est un enjouement pour luimême dans une fusion émotionnelle de l'auteur et du destinataire, libre de tout esprit critique, produite et consommée dans une gratuité du jugement comme si tout était possible ». En publicité, elle est produite, pour Jean-Claude Soulages (2006 : 106), par « un énoncé présentant une mise en cause de l'ordre rationnel du monde ou de la logique du langage qui vise à produire un état émotionnel simple de plaisir et de détente. La visée ludique ne se rattache pas en principe à une thématique spécifique et ne comporte pas de cible déterminée. Elle peut être considérée comme un pur jeu de langage ou de signification $\gg$.

2/ « La connivence critique propose au destinataire une dénonciation du faux-semblant de vertu qui cache des valeurs négatives. Elle est donc polémique (ce que n'est pas la connivence ludique) comme s'il y avait une contre argumentation implicite, car elle cherche à faire partager l'attaque d'un ordre établi en dénonçant de fausses valeurs » (Charaudeau, 2006 : 36). Selon Jean-Claude Soulages (2006), ce type de stratégie humoristique ${ }^{4}$ vise aussi, en tout cas, dans le discours publicitaire, à produire le partage de valeurs nouvelles et le rejet

\footnotetext{
${ }^{3}$ Ce texte est la refonte d'un travail mené dans le cadre d'un Programme d'action intégré entre le Centre d'analyse du discours de l'université Paris 13 et plusieurs universités espagnoles, sous la responsabilité de l'université Complutense de Madrid.

${ }^{4}$ Avec J.-Cl. Soulages, nous parlons de stratégies ludique, critique ou cynique... car, sous ces termes, seront regroupés tous les moyens sémiotiques (linguistiques, descriptifs, énonciatifs et thématiques) utilisés pour produire l'un des trois effets visés correspondants.
} 
d'anti-valeurs souvent passées de mode. Dans ce type de discours, elle comporte une cible abstraite, plus ou moins explicite, des valeurs ou des attitudes, principalement.

3/ La visée cynique : « cette stratégie vise à produire un sentiment de jubilation lié au mépris affiché de valeurs dominantes ou non, pour le seul plaisir de les mettre en cause. En aucun cas, elle ne propose « de contre-valeurs ou d'attitudes positives » (Soulages, 2006 : 108). Pour Patrick Charaudeau. (2006 : 37), « la connivence cynique a un effet destructeur. Elle est plus forte que la connivence critique car elle cherche à faire partager une dévalorisation des valeurs que la norme sociale considère positives et universelles... la vie, la mort s'en trouvent désacralisées $\gg$.

Quant à la possibilité d'une visée de dérision ${ }^{5}$ qui n'existerait en publicité - compte tenu de la législation actuelle - que sous la forme d'autodérision, on peut l'inclure dans la précédente comme une forme faible car elle attaque mal sans rien proposer, si ce n'est une connivence complice avec l'auteur (et son groupe), ce qui incite à le prendre (non sérieusement) en pitié. De la commisération empathique à la sympathie, le chemin passerait ici par l'autodérision... !

\section{Enquêtes quasi expérimentales en cours}

Au départ, on a donc voulu mettre à l'épreuve ce modèle en réception et faciliter ainsi les comparaisons entre les publicités espagnoles et françaises auprès de sujets français et espagnols. En effet, un des enjeux classiques pour une psychologie sociale de la communication ou du langage, consiste à tester le réalisme psychologique de notions élaborées par l'analyse sémantique, argumentative, ou énonciative des discours sur le modèle de la linguistique psychologique de la première époque (Chabrol, 1988 ; Chabrol, Fourquet-Courbet, Courbet, 2004 : 9). Le développement de modèles transversaux pragma-sémiotiques comme celui ci, qui lient la sémio-linguistique de l'énonciation et de l'énoncé humoristique, à travers l'étude des procédés énonciatifs et descriptifs, puis des thématiques avec les notions pragmatiques de visées de connivence, fournit des éléments pertinents pour une psycho-pragma-

\footnotetext{
${ }^{5}$ Dans un texte inédit de travail, « Humour quand tu nous tiens », P. Charaudeau pense que la dérision est intermédiaire entre la « critique » et la « cynique », car comme toutes deux elle disqualifie. Cependant, elle ne propose pas de valeurs nouvelles puisqu'elle n'argumente pas. Selon nous, elle serait donc plutôt une variété de «Cynique » modérée, car ses attaques sur les personnes en général ou les collectifs (groupes, organisations, peuples) sont si peu élaborées qu'elles se retournent facilement aussi contre son auteur (auto-dérision), sans proposer aucune autre alternative.
} 
sémiotique des discours sociaux. Naturellement, ils doivent être complétés car ce modèle ne permet pas d'articuler les visées communicationnelles aux buts d'action, et les effets visés de connivence et de plaisir supposés aux attitudes, jugements et comportements vis-àvis du produit, de la marque et des stratégies elles mêmes. En outre, les différences linguistiques, discursives et culturelles, sans parler des enjeux, capacités et connaissances variables en France comme en Espagne, devaient être repensées.

Utiliser des corpus réels et non pas reconstruits comme dans les expérimentations classiques, était indispensable ici pour rester dans la continuité des analyses de discours déjà réalisées dans le travail de l'équipe, en particulier avec Jean Claude Soulages (2006) à propos des messages publicitaires. Cette démarche excluait donc une expérimentation stricto sensu dans laquelle l'expérimentateur met à l'épreuve ses hypothèses à partir d'un matériel entièrement construit en accord avec les facteurs structurant le plan d'expérience et orientait vers une étude quasi-expérimentale. C'est-à-dire l'application d'une méthodologie expérimentale à un matériel écologique et non pas construit a priori, mais dont les caractéristiques, choisies a posteriori pour supporter le plan d'expérience, permettaient d'éprouver différentes hypothèses déduites du modèle. Dans notre esprit, cette étude devait du moins mettre à l'épreuve la pertinence des variables pragmatiques, soit les trois visées principales dans l'échantillon de publicités françaises et espagnoles dont on pouvait attendre des effets simples importants sur les jugements et attitudes, et ce malgré la diversité des produits avec leurs thématiques spécifiques et la variété des procédés énonciatifs et descriptifs.

Nous voulions aussi comparer les différences d'attitudes et jugements liées aux publicités françaises et espagnoles dans les deux pays, dans une perspective de familiarité culturelle. La familiarité des sujets en France et en Espagne, vis-à-vis des publicités des deux pays, ne pouvait être identique. En effet, les produits comme les marques, et surtout les stratégies discursives, langagières ou iconiques, connues et attendues, peuvent y différer. On avait donc fait l'hypothèse que cette familiarité jouerait aussi bien pour les mondes évoqués en référence (produit/marque) que pour les discours. En tout cas, le contrat de communication ou de lectorat (Georget, Chabrol, 2000), disponible en mémoire chez les destinataires, devait orienter les traitements en favorisant les publicités mieux connues ou plus familières culturellement. Toutefois, l'étude réalisée en parallèle en Espagne n’a pas réuni un nombre de sujets suffisants, ce qui a conduit à limiter les comparaisons aux seuls sujets français. La variable « familiarité » et le contrat de lectorat joueraient bien leur rôle, mais dans un seul pays, puisque des publicités françaises et espagnoles seraient présentées aux sujets. 
Enfin, nous voulions vérifier le degré de conscience des traitements mis en œuvre par les sujets, en particulier leur capacité de reconnaissance explicite des visées et stratégies associées. En effet, nous pouvions avoir des effets implicites et non conscients des stratégies sans reconnaissance explicite des visées ${ }^{6}$. Bref, les traitements en réception pouvaient montrer un effet sur l'évaluation du produit ou de la marque, sans pour autant aller de pair avec une reconnaissance consciente des visées, ou une appréciation explicite de l'humour utilisé dans le message.

\section{Plan d'enquête}

Une enquête a donc été réalisée sur trois groupes indépendants de 25 étudiants de la filière communication à l'université Paris 3 (75 en tout) qui ont visionné chacun quatre publicités (deux françaises et deux espagnoles traduites, facteur intra) appartenant au même type de visée. Chacun des groupes réagissait à des publicités variées correspondant, selon les experts (Soulages, 2006), à l'une des trois stratégies, ludique, critique ou cynique. La comparaison entre les groupes (facteur inter) aurait dû permettre d'apprécier l'effet du facteur stratégie lié à la visée ; la comparaison entre les publicités, d'apprécier et de tester l'effet du facteur « familiarité culturelle » (facteur intra). On a tenté de mesurer, après chaque exposition (voir questionnaire en annexe I) : l'effet de ces variables indépendantes sur des variables telles que : I'humeur déclarée $(\mathrm{Q}$ I), l'évaluation du produit (Q2), la reconnaissance explicite des visées $(\mathrm{Q} 3,4,5)$, l'appréciation de l'humour utilisé (Q6) et la compréhension de chaque message par une consigne de reformulation dirigée (Q9), suivie par une tâche de listage d'idées. On a également vérifié l'impact possible d'attitudes éventuelles positives (Q7) ou négatives (Q8) vis-à-vis de la publicité en général. À l'exception de la question 9 qui correspondait à une tâche de listage, les sujets répondaient en utilisant une échelle de Likert en sept points dont les échelons étaient formulés en accord avec le contenu de la question (voir questionnaire en annexe I). En dehors de l'effet des visées, comme on prévoyait un biais endoculturel lié au contrat de lectorat, on a également opposé, dans les traitements, les effets des messages français à ceux des espagnols. En outre, cette procédure permettait de faire des observations sur les scores de publicités isolées pour mieux comprendre les résultats globaux.

\footnotetext{
${ }^{6}$ En psychologie du langage et de la communication, on tend aujourd'hui à privilégier aussi des modèles d'influence médiatique fondés sur l'hypothèse de l'automaticité de traitements irrépressibles et non conscients des informations, liés à des attitudes implicites, et sur celle d'une recherche stratégique, non consciente, d'équilibre émotionnel et d'adaptation cognitive en fonction des buts d'action (Chabrol et al., 2004 : 16).
} 
L'organisation du plan d'expérience et le matériel - annonces utilisées, qui sont par ailleurs décrites dans le corps du texte et en annexe 3 - sont présentés dans les trois premières colonnes du tableau I. Le plan de « l'étude 》 comporte un facteur indépendant (intersujets) à trois modalités - les stratégies liées aux trois visées (ST3) - et un facteur à mesures répétées (intrasujet) à deux modalités, la familiarité culturelle (FC2) déclenchée par l'appartenance au corpus publicitaire français ou espagnol. Le plan comprend un troisième facteur, le facteur publicité (annonces publicitaires) puisque, pour chaque modalité du facteur FC, deux publicités ont été proposées. De plus, les sujets ont répondu à huit questions correspondant à huit variables dépendantes (V8). Le plan peut donc s'écrire $\mathrm{S} 25<\mathrm{ST} 3>* \mathrm{FC} 2 * \mathrm{P} 2->\mathrm{V} 8$. Cette écriture repose sur une hypothèse (au sens anglais d'assumption), celle de l'équivalence des publicités utilisées entre les stratégies alors que celles-ci sont différentes. Ainsi peut-on considérer que le croisement entre les facteurs publicité et familiarité culturelle produit un facteur publicités à quatre modalités, et que les quatre publicités étant différentes pour chacune des trois stratégies, 12 publicités ont été présentées. Les effets de ce facteur composé pourront être mis en évidence par l'analyse des effets d'interaction.

\section{Discussion des résultats quantitatifs}

Le tableau I présente les résultats descriptifs d'ensemble : les moyennes pour chacune des publicités décrites selon les modalités des facteurs du plan d'expérience à chacune des variables (questions). L'examen du tableau fait apparaitre une variabilité plus ou moins importante des publicités selon les questions. Les moyennes varient peu sur la question I (humeur). La question 2 (évaluation) témoigne de différences d'appréciation plus larges puisqu'on observe des notes moyennes allant de valeurs inférieures à 3 pour les annonces « Trina », « Plombier » et « Gazpacho » jusqu'à des notes supérieures à 5 pour « Volskwagen/ Passat » ou « Beetle ». On cherchera à voir si ces différences d'évaluation du produit sont liées à la familiarité culturelle et/ou à la stratégie. En ce qui concerne les questions 3 , 4 et 5 , portant sur l'identification de la stratégie, l'intervalle de variation apparait suffisant pour montrer que les publicités diffèrent entre elles. Cependant, un examen des notes moyennes pour une même publicité pour les trois visées révèle que certaines d'entre elles, comme par exemple « Cognac Hennessy » ou « Beetle », sont peu différenciées selon ces trois visées alors qu'on s'attendait, conformément au plan d'expérience, à ce que chaque publicité obtienne une moyenne plus élevée pour la visée à laquelle elle est rattachée. Nous reviendrons sur ce point lors des analyses ultérieures. Les deux dernières questions (7 et 8 ) - portant les attitudes positive et négative vis-à-vis de la publicité - ont produit, en moyenne, peu de différenciation selon les annonces jugées. 
Enquête psycho-langagière sur les effets de l'humour en publicité

Tableau I : Organisation du plan d'expérience et matériel utilisé (colonnes I, 2 et 3) Moyennes pour chacune des publicités et pour chaque question (colonnes 4 à $\mathrm{II}$ ) Résultats de l'analyse de variance pour la comparaison des trois visées (dernière colonne).

\begin{tabular}{|c|c|c|c|c|c|c|c|c|c|c|c|}
\hline STRATÉGIE & $\begin{array}{l}\text { FAMULARTÉ } \\
\text { CUITURELLE }\end{array}$ & PUвuCTÉ & $\begin{array}{l}\text { QUESTION I } \\
\text { HUMEUR }\end{array}$ & $\begin{array}{l}\text { Question } 2 \\
\text { EVALLATION }\end{array}$ & $\begin{array}{l}\text { QUESTION } 3 \\
\text { ID. CRITQUE }\end{array}$ & $\begin{array}{l}\text { Question } 4 \\
\text { lD. LUDIQUE }\end{array}$ & $\begin{array}{l}\text { Question } 5 \\
\text { I. CYNIQUEE }\end{array}$ & $\begin{array}{l}\text { QUESTION } 6 \\
\text { HUMOUR }\end{array}$ & $\begin{array}{l}\text { QUESTION } 7 \\
\text { ATITIUDE } \\
\text { POSTIVE }\end{array}$ & $\begin{array}{l}\text { Question } 8 \\
\text { ATTTUDE } \\
\text { NEGATINE }\end{array}$ & $\begin{array}{l}\text { COMPARAISON } \\
\text { DES TROIS VISEES }\end{array}$ \\
\hline \multicolumn{12}{|l|}{ LUDIQUE } \\
\hline & France I & $\begin{array}{l}\text { TRUMPHE } \\
\text { LINGERE }\end{array}$ & 4,72 & 4,12 & 3,32 & 4,72 & 4,08 & 3,72 & 3,76 & 2,04 & $P<.05$ \\
\hline & France 2 & $\begin{array}{c}\text { PASSAT } \\
\text { AUTOMOBILE }\end{array}$ & 4,64 & 5,36 & 4,12 & 3,60 & 3,76 & 3,28 & 3,72 & 2,08 & NS \\
\hline & ESPAGNE I & Audi A4 & 5,00 & 4,52 & 2,84 & 5,48 & 2,72 & 4,08 & 3,60 & 2,40 & $P<, 001$ \\
\hline & ESPAGNE 2 & $\begin{array}{l}\text { TRINA BOISSON } \\
\text { A L'ORANGE }\end{array}$ & 4,72 & 2,64 & 3,76 & 3,44 & 3,00 & 3,68 & 3,28 & 2,04 & NS \\
\hline \multicolumn{12}{|l|}{ CRTIQUE } \\
\hline & France I & AsprRATEUR LG & 4,40 & 4,24 & 3,92 & 3,64 & 4,24 & 4,00 & 3,44 & 2,00 & NS \\
\hline & France 2 & $\begin{array}{l}\text { PATRIMOINE } \\
\text { COM }\end{array}$ & 4,80 & 3,60 & 3,68 & 4,08 & 3,44 & 4,52 & 3,80 & 2,92 & NS \\
\hline & ESPAGNE I & Suzuki & 4,60 & 4,72 & 3,84 & 4,60 & 4,52 & 4,32 & 3,80 & 2,44 & NS \\
\hline & ESPAGNE 2 & $\begin{array}{l}\text { COGNAC } \\
\text { HENNESSY }\end{array}$ & 4,60 & 3,88 & 3,88 & 3,72 & 3,84 & 3,92 & 3,60 & 3,32 & NS \\
\hline \multicolumn{12}{|l|}{ CYNIQUE } \\
\hline & France I & $\begin{array}{l}\text { ERAM } \\
\text { CHAUSSURE }\end{array}$ & 4,92 & 3,00 & 3,84 & 4,12 & 4,40 & 4,28 & 2,92 & 2,36 & NS \\
\hline & France 2 & $\begin{array}{c}\text { BEETLE } \\
\text { AUTOMOBLIE }\end{array}$ & 4,64 & 5,08 & 3,80 & 3,72 & 3,48 & 3,92 & 3,32 & 2,8 & NS \\
\hline & ESPAGNE I & $\begin{array}{l}\text { PLOMBER } \\
\text { SLIP UVE }\end{array}$ & 4,56 & 2,84 & 2,96 & 3,84 & 3,80 & 4,24 & 3,16 & 2,28 & $P<.10$ \\
\hline & ESPAGNE 2 & $\begin{array}{c}\text { GAZPACHO } \\
\text { OZU }\end{array}$ & 5,00 & 2,96 & 2,32 & 4,04 & 3,40 & 2,68 & 2,96 & 2,76 & $P<.001$ \\
\hline
\end{tabular}

Pour l'analyse globale, on a testé l'effet de chacun des facteurs et de leurs interactions à l'aide de l'analyse de variance pour mesures répétées. Le lecteur trouvera les résultats des tests statistiques en tableau 2. L'examen des tests $(F)$ pour le facteur « stratégie » et pour chacune des huit variables (questions) n'est significatif tendanciellement (au seuil $p=.10$ ) que pour la question se rapportant à l'évaluation du produit. En bref, les produits présentés avec une stratégie ludique paraissent évalués de façon plus positive, et ceci surtout au détriment des produits présentés avec une stratégie « cynique ». On observe plusieurs tests significatifs pour le facteur « familiarité culturelle (FC) 》 ainsi que pour l'interaction entre le facteur «stratégie » et le facteur «FC », mais comme nous l'avons signalé supra, l'interprétation de ces résultats est délicate. On observe également des effets significatifs du facteur « publicité », ainsi que des effets significatifs des interactions entre ce facteur « publicité » et le facteur «familiarité culturelle », tout comme des effets significatifs des interaction entre les « facteur publicité » et le «facteur stratégie ». On observe même des effets d'interaction entre les trois facteurs St*FC*P. Ces effets simples et d'interaction du facteur publicité témoignent du fait que, pour une partie du matériel, les effets des facteurs « stratégie 》 et « familiarité culturelle » sont modifiés par le facteur « publicité ». 
Tableau 2 : Analyse de la variance pour les facteurs « stratégie », « familiarité culturelle », « publicité » et leurs interactions pour chaque question.

\begin{tabular}{cccccccc}
\hline & $\begin{array}{c}\text { Stratégie } \\
\text { (inter) }\end{array}$ & $\begin{array}{c}\text { Familiarité } \\
\text { Culturelle } \\
\text { (intra) }\end{array}$ & $\begin{array}{c}\text { Stratégie* } \\
\text { Familiarité } \\
\text { Culturelle }\end{array}$ & $\begin{array}{c}\text { Publicité } \\
\text { (intra) }\end{array}$ & $\begin{array}{c}\text { Stratégie* } \\
\text { Publicité }\end{array}$ & $\begin{array}{c}\text { Familiarité } \\
\text { Culturelle* } \\
\text { Publicité }\end{array}$ & $\begin{array}{c}\text { Stratégie* } \\
\text { Familiarité } \\
\text { Culturelle* } \\
\text { Publicité }\end{array}$ \\
\hline HUMEUR & $\mathrm{ns}$ & $\mathrm{ns}$ & $\mathrm{ns}$ & $\mathrm{ns}$ & $\mathrm{ns}$ & $\mathrm{ns}$ & $\mathrm{Ns}$ \\
\hline EVALUATION & $\mathrm{P}<.10$ & $\mathrm{P}<.00 \mathrm{I}$ & $\mathrm{P}<.001$ & $\mathrm{~ns}$ & $\mathrm{P}<.001$ & $\mathrm{P}<.001$ & $\mathrm{P}<.01$ \\
\hline ID CRTIQUE & $\mathrm{ns}$ & $\mathrm{P}<.001$ & $\mathrm{P}<.05$ & $\mathrm{~ns}$ & $\mathrm{P}<.05$ & $\mathrm{Ns}$ & $\mathrm{Ns}$ \\
\hline ID LUDIQUE & $\mathrm{ns}$ & $\mathrm{ns}$ & $\mathrm{ns}$ & $\mathrm{P}<.01$ & $\mathrm{P}<.01$ & $\mathrm{Ns}$ & $\mathrm{P}<.10$ \\
\hline ID CYNIQUE & $\mathrm{ns}$ & $\mathrm{P}<.05$ & $\mathrm{P}<.05$ & $\mathrm{P}<.01$ & $\mathrm{P}<.10$ & $\mathrm{Ns}$ & $\mathrm{Ns}$ \\
\hline HUMOUR & $\mathrm{ns}$ & $\mathrm{ns}$ & $\mathrm{ns}$ & $\mathrm{ns}$ & $\mathrm{ns}$ & $\mathrm{Ns}$ & $\mathrm{Ns}$ \\
\hline $\begin{array}{c}\text { ATTITUDE } \\
\text { POSITINE }\end{array}$ & $\mathrm{ns}$ & $\mathrm{ns}$ & $\mathrm{ns}$ & $\mathrm{ns}$ & $\mathrm{ns}$ & $\mathrm{P}<.05$ & $\mathrm{Ns}$ \\
\hline $\begin{array}{l}\text { ATTITUDE } \\
\text { NÉGATIVE }\end{array}$ & $\mathrm{ns}$ & $\mathrm{P}<.10$ & $\mathrm{~ns}$ & $\mathrm{P}<.01$ & $\mathrm{P}<.01$ & $\mathrm{Ns}$ & $\mathrm{Ns}$ \\
\hline
\end{tabular}

L'impossibilité de conclure à un effet systématique du facteur stratégie et le caractère « erratique » des effets simples et d'interaction du facteur 《 familiarité culturelle », et surtout du facteur « publicité », nous conduisent à nous interroger sur les catégories utilisées par les sujets. Rappelons que ce matériel est écologique et que la classification selon les stratégies a été faite par les analystes-experts, au terme de discussions?. Si, en analyse de variance, on considère en général dans le cadre de l'inférence fréquentiste qu'un résultat non significatif n'est pas informatif, on peut considérer que cette conclusion est le reflet des éléments entrant dans le calcul du test et, en particulier, le fait que le dénominateur du test est sensible à la fois à l'effectif de l'échantillon et à sa dispersion. On peut se permettre de faire l'hypothèse qu'il existe une variabilité dans la réception des publicités, et que l'identification de la stratégie qu'elles sont censées représenter n'est pas univoque chez tous les sujets de chaque groupe et n'est pas identique à l'identification a priori posée par les experts-analystes.

On peut faire l'hypothèse qu'une « variabilité interindividuelle » pourrait aussi exister entre les sujets de chacun des groupes. Ces différences interindividuelles de perception des publicités pourraient expliquer, en partie, l'absence d'effet du facteur « stratégie », dans la mesure où l'étude d'un effet moyen, agrégeant des jugements individuels, n'aurait pas beaucoup de pertinence dans le cas où les jugements des sujets différeraient non pas quantitativement, mais qualitativement. De plus, on ne peut écarter l'idée que les jugements des sujets pourraient différer également de celui des experts, ce qui mettrait en défaut la fiabilité du plan d'étude, puisque les modalités définies a priori par les experts ne seraient pas identiques à celles utilisées a posteriori par les sujets. ${ }^{8}$ Certes, dans la

\footnotetext{
${ }^{7}$ Les discussions ont eu lieu dans le groupe de travail franco-espagnol « Picasso », puis dans l'équipe française parmi les collaborateurs de cette livraison de Questions de communication. L'accord a été obtenu pour la très grande majorité des publicités évoquées ici, sauf une « Triumph », classée ludique ou cynique.

${ }^{8}$ Compléter le travail des experts par une procédure d'évaluation avec des juges pris dans la même population et entraînés, sera utile à l'étape suivante.
} 
mesure où nous n'avons pas les moyens de contrôler l'indépendance des variables, une telle réflexion ne peut être considérée que comme exploratoire. II est malgré tout assez probable que les sujets sont sensibles à l'effet de connivence ludique, critique ou cynique, mais en interaction avec quelques-unes des variables non mesurées, telles l'appréciation de la marque, du produit, ou encore l'attitude face au genre discursif publicitaire ou à l'égard des thématiques (féministe en particulier). La proximité en termes de connaissance et de goût avec des procédés sémantiques énoncifs (loufoque, absurde et paradoxal), ou énonciatifs (sarcastique, ironique ou parodique), peut aussi intervenir (Charaudeau, 2006). Cela dit, à partir des données et pour commencer à tester ces conjectures, nous pouvons au moins évaluer l'impact de la reconnaissance explicite des stratégies, soit les réponses aux trois questions 3, 4 et 5 portant sur la reconnaissance explicite de la visée (critique, ludique et cynique) et les comparer aux résultats du listage d'idées. Sous l'hypothèse d'une reconnaissance de la visée en accord avec le plan d'étude, on s'attend à ce qu'une des moyennes à ces trois questions diffère significativement des deux autres, et sous l'hypothèse de la cohérence avec le plan a priori, que cette moyenne soit celle qui corresponde à la modalité retenue pour la publicité. Nous avons testé ces hypothèses en utilisant l'analyse de variance pour des mesures répétées. On a conduit des analyses spécifiques en travaillant sur chaque publicité séparément (les résultats sont présentés dans la dernière colonne du tableau I).

L'hypothèse I (existence d'une différence de moyenne pour au moins une des stratégies) peut être retenue pour quatre publicités («Triumph», $p=.05 ;$ « Audi », $p=.001 ;$ «Plombier », $p=.10 ;$ « Gazpacho », $p=.00$ I). On a donc poursuivi l'analyse pour tester la seconde hypothèse en comparant la moyenne de la publicité définie a priori (stratégie à laquelle elle est rattachée dans le plan) aux moyennes des deux autres questions portant sur les stratégies. L'hypothèse d'une reconnaissance correcte de la visée peut être retenue pour « Triumph » (moyenne ludique $>$ moyenne critique et cynique $p<.01$ ) et pour «Audi » (moyenne ludique > moyenne critique et cynique $p<.00 \mathrm{I}$ ), mais l'examen des moyennes montre que «Plombier » est moins identifié comme cynique $(m=2.86)$ que comme ludique $(m=3.84)$ ou critique $(m=3.80)$ (significatif à $p<.05)$ et que «Gazpacho » est plus identifié comme ludique $(m=4.04)$ que cynique $(m=3.40)$.

On peut donc conclure que huit des douze publicités ne font pas l'objet d'un consensus sur la reconnaissance d'une des trois stratégies, et que deux publicités sont rattachées à une stratégie qui n'est pas celle qui était définie a priori. Seules deux publicités (« Triumph » et « Audi ») voient leurs visées identifiées, majoritairement en conformité avec leur identification a priori par les experts. Ces résultats, en particulier l'absence d'une identification claire à l'une des trois visées pour huit des publicités, laissent 
penser qu'il existerait peut-être une variabilité interindividuelle dans la réception de ce matériel, du moins si l'on suppose que l'absence d'une reconnaissance explicite est un premier indice, certes insuffisant, du processus à l'œuvre dans le traitement. Pour tenter de mettre en évidence cette variabilité, il était souhaitable de revenir aux sujets et à leurs productions à propos de chacune des publicités. Nous avons donc sélectionné, pour chacune des publicités, des sujets représentatifs de positions extrêmes, soit qu'lls n'aient « pas du tout » reconnu une visée donnée pour la publicité présentée (choix de la note I de l'échelle), soit, au contraire qu'ils aient « tout à fait » reconnu la visée pour cette publicité (note 7 de l'échelle).

\section{Une nouvelle voie : I'analyse qualitative du listage d'idées}

Un premier commentaire rapide peut souligner la difficulté des sujets à catégoriser les stratégies correspondant aux visées puisque, sur 25 sujets par groupe, une majorité de sujets se réfugient dans des réponses proches de la moyenne pour les publicités classées « Ludique », dont seuls quelques-uns ressentent clairement la visée supposée (Ludique fort : «Audi » puis « Triumph »). Pour cette analyse, nous avons identifié et regroupé les sujets ayant donné des réponses extrêmes - faibles (échelons I et 2) ou fortes (échelons 6 et 7) - aux questions portant sur la reconnaissance explicite des visées $(Q 3,4,5)$. On trouvera ces informations dans le tableau $A$ en annexe 3. Examinons maintenant quelques pensées associées aux messages publicitaires à la suite du « listage d'idées » demandé par certains de ces sujets présentant des réponses extrêmes:

Exemple I : La publicité de lingerie « Triumph » (classée par experts en Ludique).

« La saison de la chasse sera toujours trop courte 》, avec des sous-vêtements posés sur deux coussins de soie comme un trophée ou des armes.

- Orientation cynique : les sujets $n^{\circ} 3$ et 6 ou 8 et 9 la notent comme fortement « cynique » et déclarent de façon concordante :

3 « Faire passer de la lingerie comme un bien de consommation (comme si la femme devait être chassée !) nul !».

6 « La lingerie donne envie aux hommes de partir « à la chasse » aux femmes. La femme est un gibier $\gg$.

8 « II s'agit d'une publicité de marque de sous-vêtements et la gente masculine est pointée du doigt ».

9 « Les femmes sont des gibiers ».

- Orientation critique-ludique : les sujets 18, 19 et 22, en revanche, la notent comme « ludique » et déclarent de façon plus distanciée, si ce n'est ironiquement «féministe »: 
Enquête psycho-langagière sur les effets de l'humour en publicité

18 « Sors dans la rue en sous-vêtements, tu es sûre de ne pas rentrer toute seule. Avec la lingerie « Triumph » tu es sûre de garder ton homme 》.

19 «Qu'en lingerie Triumph, une femme se sent prête à conquérir tous les hommes 》.

22 « Le printemps, saison des amours (dans le règne animal principalement) ne dure que peu de temps, alors dépêchez-vous! ».

Le message est donc bien perçu de façon profondément différente et ceci fait ressortir son caractère polyvalent. Le rapprochement convenu avec le monde animal peut être ressenti comme fortement dévalorisant, en particulier pour celles qui ont des attitudes « féministes » marquées et qui l'interprètent comme faisant de la femme : l'objet-victime, tandis qu'il en va tout autrement pour celles qui la constituent comme sujet de l'activité « cynégétique ».

Exemple 2 : La publicité « L'aspirateur » (classée par experts en « critique »).

« Non Jonathan, ceci n'est pas une cafetière. Mais le récipient à poussière de notre aspirateur sans sac », avec un athlète au corps huilé qui brandit ce récipient comme une coupe ».

La répartition presque équilibrée dans toutes les possibilités de classement étonne. Il est ressenti comme fortement « critique » par quatre personnes et fortement « cynique » par six sujets :

- Orientation critique :

47 «Que le produit n'est plus seulement réservé aux femmes».

48 « Cet aspirateur peut être utilisé aussi bien par des hommes que des femmes. \|l faut changer les mentalités ».

49 «Que l'homme doit plus s'investir dans les tâches quotidiennes. Le ménage est réservé aux femmes en général, donc les femmes sont plus intelligentes ».

50 « Cet aspirateur a l'air facile d'utilisation... Les hommes n'ont plus d'excuses pour ne pas le passer. Les femmes sont incitées à ne pas rester dans leur carcan social ».

- Orientation cynique :

27 «Que même les hommes, aussi bêtes soient-ils, peuvent passer l'aspirateur ».

33 « Un homme nu tient un sac d'aspirateur en pensant que c'est une cafetière. Heureusement, sa femme le reprend $\gg$.

42 « L'homme n'est pas à jour sur les nouvelles techniques ménagères ».

Mais 47, 49, 50 notent fort aussi bien en critique qu'en cynique : l'ambivalence des trois derniers sujets ressort clairement. Elles approuvent l'orientation « Critique » supposée par elles « féministe » et notent fort en Critique mais ressentent un certain malaise à voir ainsi disqualifier l'homme, ce qui les incitent aussi à noter fortement en cynique.

Exemple 3 : La publicité espagnole des automobiles « Suzuki » (classée par experts en critique).

« Les statistiques démontrent que nous sommes plus sûres quand nous conduisons. Qui sait... parce que nous autres pensons quand nous achetons » avec une jeune femme debout, tenant un jeune enfant dans les bras, bien en équilibre, en pantalon et jambes écartées ». 
L'on voit une même personne ( $\left.n^{\circ} 33\right)$ noter fortement le message comme à la fois critique, ludique et cynique :

33 « L'arrivée d'une voiture destinée aux femmes. Le domaine des voitures n'est plus réservé aux mecs ».

Commentaire qui fait ressortir seulement l'orientation «Critique » dans sa pensée déclarée!

Les $28,34,36$ et 47 , notent fortement « cynique » tout en posant un commentaire « critique » (28 et 34$)$ alors que 36 et 47 réagissent à la disqualification des hommes mais en atténuent beaucoup la force :

28 « Elle veut démontrer que c'est une voiture qui n'est pas réservée qu'aux femmes 》.

34 « Les femmes sont plus intelligentes que les hommes car les femmes veulent de la qualité donc elles achètent cette voiture $»$.

36 « On a voulu vendre une voiture aux femmes alors on a fait une petite blague sexiste, bon enfant $\gg$.

47 « Elle veut démontrer que c'est une voiture qui n'est pas réservée qu'aux femmes $\gg$.

Exemple 4 : La publicité pour le Cognac (classée par experts en critique).

« Un bon cognac s'associe à l'image d'un homme mûr fumant un havane » avec une jeune femme élégante, cheveux courts en veste et pantalon ».

Cette publicité montre encore une belle dispersion d'où ressort l'hésitation des sujets 27, 33 et 49 qui notent fortement en critique et ludique et même en cynique $(27,33)$ :

27 « Le publicitaire essaye de persuader que le produit présenté est le meilleur de tous » (peu ludique, cynique ? !).

33 « Le publicitaire est provoquant (paradoxe entre le slogan et l'image). II tente de casser les traditions » (orientation plus critique que ludique?).

49 «Que tout ce qui nuit à l'humain ne doit pas être réservé aux hommes. Le plaisir dangereux est aussi excitant pour les femmes » (orientation cynique ?).

Les sujets 44, 46 notent faiblement partout avec pourtant des pensées clairement d'orientation critique :

44 « Une femme fatale peut boire du cognac ! ça casse les "a priori" ».

46 « Une femme aux allures d'hommes casse l'image de la femme fébrile grâce à la présence d'un cigare et d'une bouteille de cognac ».

Tandis que 40 note faiblement critique et cynique avec une orientation plutôt cynique :

40 «Que l'alcool, c'est pas réservé aux hommes et que tout le monde peut être un salaud décomplexé ».

Au passage, il faut noter la grande sensibilité des sujets féminins à la forme de dérision utilisée. Le message « Suzuki » (« Les statistiques démontrent que nous sommes plus sûres quand nous conduisons, qui sait... parce que nous autres pensons quand nous achetons ») est noté

\footnotetext{
${ }^{9}$ Cette distorsion ente la notation et la pensée déclarée interroge. Les sujets sanctionnent implicitement la disqualification de l'homme, mais approuvent explicitement la promotion de la femme dans un domaine de compétence, l'automobile, jugé encore souvent comme masculin.
} 
Enquête psycho-langagière sur les effets de l'humour en publicité

comme plus cynique que celui consacré au cognac qui ne fait qu'évoquer un temps où seul les hommes mûrs en buvaient avec un cigare, ce qui n'instaure aucune dérision ou disqualification.

Exemple 4 : La publicité « Eram » (classée par experts en cynique).

« Aucun corps de femme n'a été utilisé dans cette publicité » avec un homme nu, jeune, mince et poilu avec un bouc et des chaussures de femme, le message pouvait être assez bien reconnu comme cynique sans exclure d'autres lectures.

II est en effet noté fortement comme tel par 6 sujets sur 24, ce qui est à souligner :

- Orientation cynique :

52 « Réponse du berger aux "bergères", le publicitaire joue l'enfant sage. Le message est clair : si on ne peut pas faire ce que l'on veut du corps des femmes sur les affiches autant prendre des hommes, tout ou rien ? ».

56 « Cette pub nuit gravement à l'éthique ».

57 « $Y$ a des gens nus partout même pour des pompes, c'est moche ! Rien à comprendre de plus ».

74 « Que les féministes sont des emmerdeuses ».

- Orientation critique :

63 « La publicité nous montre qu'il n'est pas nécessaire d'avoir une femme nue dans une publicité ».

72 « La publicité prouve qu'une femme nue n'est pas obligatoire en publicité ».

Toutefois 56 et 63 ont noté dans le même temps fortement ludique, ce qui surprend pour 56, attentif à l'éthique. 63 est, lui, plus cohérent car noter ludique et fortement critique le même message n'est pas si contradictoire. 62 note lui aussi fortement ludique avec une déclaration clairement « critique », équivalente à 63 et 72 . En somme, si le sujet apprécie l'appariement de l'orientation stratégique (critique) et du procédé paradoxal utilisé, il en ressentirait un plaisir euphorique qui le porterait à juger aussi ludique ce message.

75 déclare « Eram fait un pied de nez aux féministes en nous invitant à penser que ce ne sont pas toujours les corps de femmes qui sont exploités dans les pubs. Mais espère-t-elle une retombée directe en terme de ventes ? Plus pour leur réputation ».

75 attribue à Eram une visée « critique » complexe puisqu'il saisit la charge contre les «féministes » mais en inverse la portée. En somme, si la pub fait la même chose du corps des hommes que de celui des femmes, elle rétablit une égalité de traitement qui rend caduque la principale revendication des féministes, tout en l'intégrant pour la dépasser ! Au passage, ce sujet pressent une distinction importante, la visée vers le produit ou vers la marque. Ce message Eram ne met pas en valeur les qualités des chaussures, ni de celles qui les portent. II promeut seulement les qualités de la Marque-Annonceur, son ethos de provocateur subtil.

Exemple 5 : Les slips féminins espagnols « uve » (classée par experts en cynique).

«Malheureusement tout le monde ne peut pas porter nos slips », avec un dos de plombier espagnol obèse exhibant le début de son postérieur dans l'effort ».

La procédure « sarcastique » presque grotesque et le paradoxe des contraires réunis sont sans doute fort éloignés des contrats de lectorat habituels en France pour ce type de produit. L'évaluation du produit est faible et sa marque inconnue aggrave sans doute les choses. Toutefois et à nouveau, on trouve des notations fortes en ludique et cynique (4 dans chaque) : 
62 « Beaucoup d'humour mais j'ai pitié quand même pour les personnes obèses car je crois pas qu'ils le prennent très bien » (orientation cynique et ludique à la fois ).

56 " Ça c'est pour prôner le "sexisme" à travers les moyens de communication et la pub n'échappe pas à la règle » (orientation cynique)

57 « C'est moche ! » (orientation cynique pointée).

\section{Conclusion}

Ce long inventaire suggère plusieurs constats. D'abord, les sujets ont souvent du mal à faire un choix quand bien même décident-ils de noter fortement. Or, au-delà d'erreurs possibles d'interprétations des questions, liées à une formulation insuffisamment explicite, on peut penser que certains sujets ne semblent pas parvenir à décider de la prédominance d'une seule visée. Ainsi une visée critique ne sera-t-elle pas reconnue comme unique mais liée à une visée cynique parce que, par exemple, ces personnes ressentent très fortement les procédures de disqualification en particulier de « l'homme » avec une thématique « féministe » ou « sexiste », et rejettent sans doute une procédure « sarcastique » qui multiplie la force de la dérision. Les étudiantes françaises préfèreraient l'ironie ou la parodie au sarcasme et les connivences fondées sur le « ludique » ou le « critique » si la dérision n'est pas trop dirigée sur une cible humaine. Ensuite, les pensées déclarées peuvent souvent être en désaccord avec les notations. Ce manque de cohérence n'est pas surprenant en soi, mais sa répétition indique probablement une ambivalence des sujets face à la polyvalence des messages. Ils décideraient de faire apparaître l'une des dimensions et non plusieurs, parce qu'il est moins incohérent de noter plusieurs fois fortement le même objet que de déclarer à son propos des pensées très différentes. En outre, de façon générale, les notations faibles ont rassemblé plus de sujets que les fortes (si ce n'est pour « Triumph » et « Audi ») : 63 sujets contre 28 en « critique », 43/28 en 《ludique » et 60/39 en « cynique ». Ceci renforce l'idée d'une indécision des sujets que la diversité des pensées déclarées confirme, d'autant que les mêmes sujets notent souvent faiblement dans plusieurs catégories le même message. Enfin, remarquons que la très grande majorité des notes se situent dans les zones moyennes, ni faible, ni forte. À peine un tiers des sujets se positionne sur les extrêmes pour les reconnaissances des visées « critique » et « cynique », et seulement le quart pour la « ludique » qui exige sans doute plus de travail interprétatif. En somme, cette prudence renforce à nouveau l'idée que la reconnaissance est loin d'être aisée car jamais univoque dans ces processus. Notons encore que fort peu de sujets expriment explicitement une pensée cynique de façon positive. Ils la désapprouvent dans le listage d'idées, sauf à de très rares exceptions. Ceci ne signifie pas qu'implicitement, il en aille ainsi ou autrement. De fait, nous ne disposons, ici, que de peu de moyens pour examiner cette question. 
Si l'on accorde un crédit à ces premiers résultats qualitatifs, nous pourrions commencer à penser maintenant que l'hypothèse de la variabilité inter-individuelle dans le traitement des publicités est plausible à l'intérieur d'un même groupe socialement défini. Toutefois, des expérimentations véritables auprès de sujets masculins et féminins ${ }^{10}$, et des focus groupes pour approfondir l'interprétation des résultats, seraient nécessaires pour confirmer ces conjectures qui complexifient les recherches appliquées et fondamentales sur les traitements et effets des messages humoristiques, en particulier dans les médias. II faudra naturellement distinguer clairement les effets comportementaux attendus négligés ici, ou du moins l'intention comportementale des effets sur les jugements, attitudes et affects examinés ici. Quoi qu'il en soit, il faudra bien prendre acte de la complexité d'un message humoristique. Au niveau énoncif, sa structure sémantique doit être reconstruite au prix de nombreuses inférences, car les procédés descriptifs (loufoque, absurde ou paradoxal) déplacent et transgressent les logiques qui soustendent nos connaissances du monde (Charaudeau, 2006). Ce travail interprétatif implique donc des connaissances, des capacités et des motivations non négligeables, en particulier les énoncés « ludiques » très consommateurs d'attention, d'inférences étayées sur des connaissances intertextuelles, de capacités à déchiffrer des figures et des tropes et à les insérer dans l'interprétation. Bref, des motivations, des connaissances et des capacités sont requises pour traiter ces messages, et ceci ne peut qu'augmenter les différences entre individus dans un groupe socialement homogène.

Par ailleurs, au niveau énonciatif, les procédés « ironique » et « sarcastique » dirigent vers la cible concrète (personne, animal, organisation), ou abstraite (valeurs, catégorie sociale, groupe), des attaques qui la disqualifient plus ou moins fortement. Ces procédés sont ressentis comme plus ou moins chargés d'agressivité, mais celle-ci sera ressentie différemment en fonction de l'orientation attitudinelle (pro/anti ou neutre) du sujet récepteur. L'appréciation de l'humour est donc suspendue à une convergence, au moins relative (pro ou neutre), des attitudes entre énonciateur et énonciataire. Lorsque tel n'est pas le cas, il y a rupture du « pacte humoristique » qui consiste normalement à donner crédit à l'auteur, d'une position d'énonciation « non sérieuse » qui suspend les devoirs de sincérité, de pertinence, de vérité, en installant la possibilité d'une connivence. À l'inverse, lorsque l'énonciateur met en cause les croyances et les attitudes du destinataire, liées aux valeurs évoquées, surtout si on les suppose fortes, ce dernier peut suspendre le pacte, en pensant que

\footnotetext{
${ }^{10}$ Par commodité et au vu de moyens limités, nous avons d'abord neutralisé la variable de « sexe » en n'interrogeant que des sujets féminins. II n'est pas impossible que celle-ci, sous la forme d'un facteur de « genre sexuel » intervienne mais, comme toujours, celle-ci n'est pas explicative, mais descriptive, sans parler de causalité !
} 
l'énonciateur est plus « sérieux » qu'il ne veut le paraître, et en posant sa responsabilité d'être du monde, il rompra la complicité et rejettera la connivence attendue. Si l'on accorde au moins une part de vérité à ces positions, les résultats constatés plus haut n'étonneraient plus. Même dans un groupe socialement homogène, les conditions requises pour la production d'un même effet positif convergent, à la fois au plan des conduites attendues (achat), des jugements et des affects ressentis, d'un énoncé publicitaire humoristique totalement traité, sont telles, que celui-ci semble presque improbable. Ceci devrait diminuer l'optimisme des annonceurs.

Enfin, cette étude suggère que, pour saisir les effets des stratégies humoristiques en publicité, il faudra déployer un cadre conceptuel psychosociologique, pragmatique et sémiotique à la fois. Par exemple, on tentera de prouver les effets positifs, au moins sur les jugements, attitudes, et intentions comportementales, d'une corrélation positive entre la connivence ludique, des objets thématiques peu « investis » par des indices d'attitudes pro/anti vis-à-vis des objets-valeurs évoqués par la thématique associée au produit" et des liens privilégiés avec le procédé « ironique » et l'incohérence 《l loufoque ». Cette constellation sémio-pragmatique conviendrait bien à des personnes dotées de bonnes connaissances et capacités d'inférence, orientées vers une certaine attitude de réactance envers la publicité en général, à laquelle elles dénieraient en tout cas le droit de promouvoir des valeurs ou de les attaquer et assez motivées pour traiter de façon approfondie et « centrale » les messages pour eux-mêmes en tant que jeu sur le langage. Les sujets à fort besoin de cognition pourraient préférer ce type d'humour aux autres parce qu'il exige, s'il est traité complètement, plus d'efforts et n'a aucune prétention au plan de l'argumentation sérieuse. Cette forme d'humour devrait souvent convenir aux marques prestigieuses et aux produits jugés évidemment utiles qui, au fond, n'auraient ni à convaincre ni à séduire. De même, on devrait mettre à l'épreuve l'efficacité des liens supposés entre la connivence critique et le soutien fort à des objets d'attitude conformes aux normes et valeurs émergentes dans la contemporanéité et la disqualification des anciennes valeurs tombées en désuétude, avec une certaine inclination pour le procédé « sarcastique » et des liens étroits avec les incohérences absurdes ou paradoxales. Cette constellation sémio-pragmatique devrait convenir à des personnes qui s'investissent dans les confrontations idéologiques contemporaines bien médiatisées ou, du moins, toujours au plus près des évolutions du « penser correct » au plan groupal et sociétal. Les sujets à « self monitoring » élevé pourraient être plus sensibles que les autres à cette forme d'humour ${ }^{2}$. Sous la forme d'une légère dérision, elle est souvent associée à des marques jugées moyennement prestigieuses ou des produits pensés relativement utiles. On devra vérifier l'efficacité de cette relation.

\footnotetext{
"L'humour, en particulier « ludique », utilise des thématiques sociales et affectives très souvent sans rapport du moins immédiat avec les qualités du produit.

12 II n'est pas du tout impossible que l'humour ne soit pas ou très peu traité par une partie de ces personnes à haut self monitoring
} 
Enfin, pour la connivence cynique, en relation avec le procédé « sarcastique » et associée aux incohérences absurdes ou paradoxales, on attendra des rapports producteurs d'effets positifs, en particulier chez les sujets qui trouvent de l'attrait pour toutes les formes de disqualification et dérision des valeurs et normes sociétales contemporaines, conformistes ou novatrices, y compris une autodérision du discours publicitaire médiatisé par lui-même. Elle pourrait séduire les personnes tentées par le repli pessimiste, le rejet ou la rébellion groupale. Ces sujets trouveraient dans cette forme d'humour un expédient pour exprimer, plus ou moins sérieusement, une résistance aux évolutions actuelles de notre société, de façon, elle aussi, à la limite auto-dérisoire. Cette forme attirerait surtout l'attention sur des marques souvent peu prestigieuses et/ou des produits jugés peu utiles.

On l'aura compris, nous avons posé la conjecture de la variabilité interindividuelle de façon transitoire ou intermédiaire pour décrire les premiers résultats. Cela convenait à ce qui se passait dans un groupe socialement homogène, mais nous pouvons maintenant proposer un ensemble d'hypothèses générales avec des variables qui associent des facteurs psychosociaux et sémio-pragmatiques, dont il faudra tester point par point les applications. Naturellement, comme les formes et variétés d'humour diffèrent beaucoup et peuvent demander des connaissances et efforts cognitifs importants et des orientations attitudinelles précises, les processus de traitement approfondis ou centraux requis impliqueront souvent des conditions difficiles à réunir, d'autant que ces énoncés sont susceptibles d'interprétations plurielles (ambivalence, polyvalence, équivoque référentiel). Cela ne peut que favoriser des traitements superficiels ou périphériques très divers, qui pourraient redonner aux conjectures de « variabilités inter et même intra individuelles », un certain attrait dans le futur.

\section{Références}

Cacioppo J.T., Petty R. E., 1982, « The need for cognition », Journal of Personality and Social Psychology, 42, pp. I16-131.

Chabrol Cl., 1988, « Le lecteur. Fantôme ou réalité. Étude des processus de réception », pp. |61-183, in : Charaudeau P., éd., La presse. Produit, production, réception, Paris, Didier-Érudition.

Chabrol Cl., Fourquet-Courbet M.-P., Courbet D., 2004, « Introduction », Questions de communication, 5, pp. 5- I8.

Charaudeau P., 2006, «Des catégories pour l'humour », Questions de communication, 10, pp. 19-41.

Georget P., 2004, « Stratégie publicitaire et variable de personnalité, de contexte et de contrat », Questions de communication, 5, pp. 69-82.

Georget P., Chabrol Cl., 2000, « Traitement textuel des accroches et publicités argumentées », Revue internationale de psychologie sociale, 13 (4), pp. 17-50. 
Soulages J.-Cl., 2006, « Les stratégies humoristiques dans le discours publicitaire », Questions de communication, 10, pp. 103-1 18.

Smith S. M., 1993, « Does humor in advertising enhance systematic processing ? », pp. 155-158, in : McAlister L., Rothschild M. L., eds, Advances in consumer research, 20, Provo UT, Association for Consumer Research.

Snyder M., DeBono K. G., 1985, « Appeals to image and claims about quality: understanding the psychology of advertising », Journal of Personality and Social Psychology, 49, pp. 586-597.

Wegener D. T., Petty R. E., Smoak. D., Fabrigar L. R., 2004, « Multiple Routes to Resisting Attitude Change », pp. 13-38, in : Knowles E. S.. Linn J. A., eds, Resistance and Persuasion, Mahwah NJ, Lawrence Erlbaum Associates.

Zhang Y., 1996, « The effect of humour in advertising : an individual-difference perspective », Psychology and Marketing, 13, pp. 53।-545. 
Enquête psycho-langagière sur les effets de l'humour en publicité

Annexe I : Questionnaire.

I/ En ce moment vous estimez que votre humeur est :

$\begin{array}{llllllll}\text { I } & 2 & 3 & 4 & 5 & 6 & 7 & \\ \text { négative } & & & & & & & \text { positive }\end{array}$

2/ Le produit présenté est un produit de qualité :

$\begin{array}{lrrrrrrrr}\text { I } & 2 & 3 & 4 & 5 & 6 & 7 & \\ \text { PAS DU TOUT } & & & & & & & \text { TOUT À FAIT }\end{array}$

3/ Ce message tente de soutenir, de façon plaisante des valeurs sociales modernes :

$\begin{array}{lcccccccc}\text { I } & 2 & 3 & 4 & 5 & 6 & 7 & \\ \text { PAS DU TOUT } & & & & & & & \text { TOUT A FAIT }\end{array}$

4/ Cette publicité invite surtout à faire jouer notre imagination en mettant en cause la réalité et le langage :

$\begin{array}{lrrrrrrr}\mid & 2 & 3 & 4 & 5 & 6 & 7 & \\ \text { PAS DU TOUT } & & & & & & \text { TOUT À FAIT }\end{array}$

5/ Ce message tend surtout à disqualifier, de façon plaisante, des personnes et/ou des valeurs :

$\begin{array}{lrrrrrrr}\text { I } & 2 & 3 & 4 & 5 & 6 & 7 & \\ \text { PAS DU TOUT } & & & & & & \text { TOUT À FAIT }\end{array}$

6/Vous avez apprécié l'usage de l'humour dans cette publicité :

$\begin{array}{lrrrrrrrr}\text { I } & 2 & 3 & 4 & 5 & 6 & 7 & \\ \text { PAS DU TOUT } & & & & & & \text { TOUT À FAIT }\end{array}$

7/ A votre avis, la publicité est un moyen d'information somme toute efficace et réaliste sur les biens et les services offerts sur le marché :

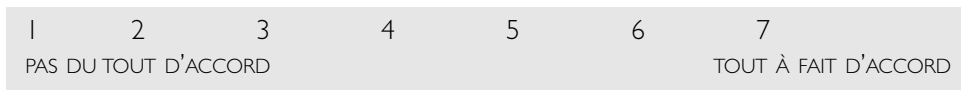

8/ A votre avis, la publicité est une forme de propagande qui oriente de façon insidieuse les désirs et les besoins des consommateurs, malgré eux.

$\begin{array}{lrrrrrr}\text { I } & 2 & 3 & 4 & 5 & 6 & 7 \\ \text { PAS DU TOUT D'ACCORD } & & & & \text { TOUT À FAIT D'ACCORD }\end{array}$

9/ Si vous deviez expliquer cette publicité à quelqu'un de votre entourage, que diriez-vous que le publicitaire essaye de faire comprendre. Écrivez ci dessous les trois idées principales, que selon vous, il voulait transmettre :

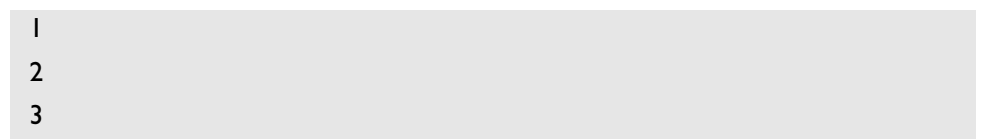


Annexe 2 : Description des publicités non évoquées dans le texte.

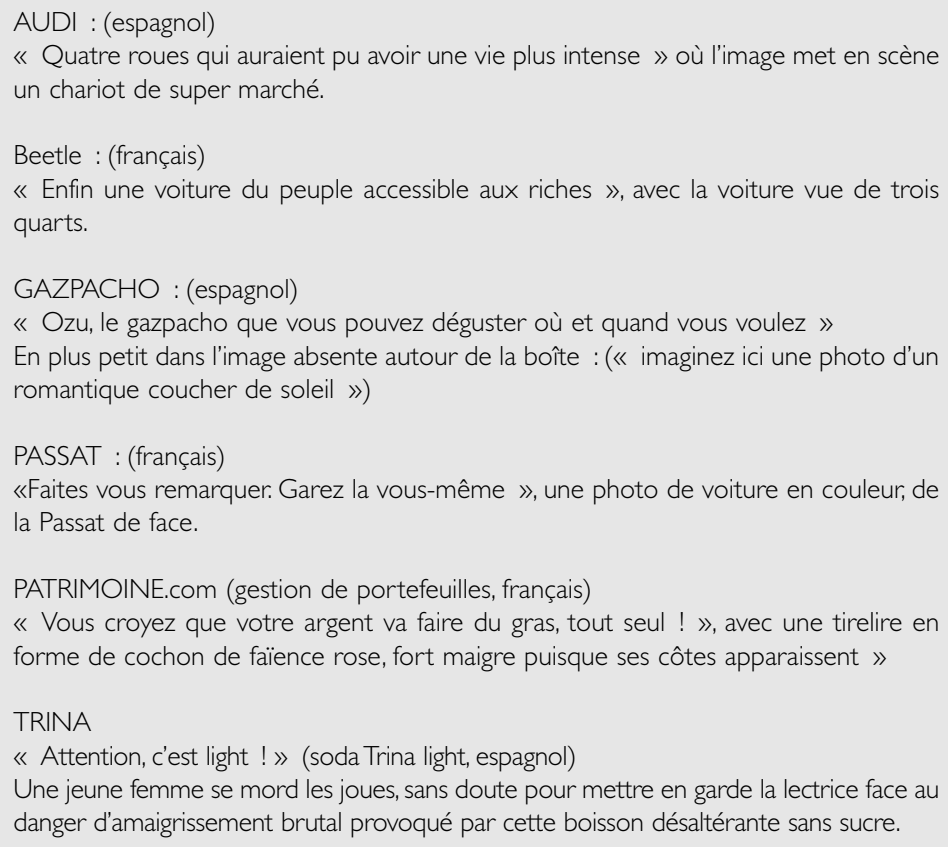

«Quatre roues qui auraient pu avoir une vie plus intense » où l'image met en scène un chariot de super marché.

Beetle : (français)

« Enfin une voiture du peuple accessible aux riches », avec la voiture vue de trois quarts.

\section{GAZPACHO : (espagnol)}

«Ozu, le gazpacho que vous pouvez déguster où et quand vous voulez »

En plus petit dans l'image absente autour de la boîte : (« imaginez ici une photo d'un romantique coucher de soleil »)

\section{PASSAT : (français)}

«Faites vous remarquer. Garez la vous-même », une photo de voiture en couleur, de la Passat de face.

\section{PATRIMOINE.com (gestion de portefeuilles, français)}

« Vous croyez que votre argent va faire du gras, tout seul ! », avec une tirelire en forme de cochon de faïence rose, fort maigre puisque ses côtes apparaissent »

TRINA

« Attention, c'est light! ! (soda Trina light, espagnol)

Une jeune femme se mord les joues, sans doute pour mettre en garde la lectrice face au danger d'amaigrissement brutal provoqué par cette boisson désaltérante sans sucre.

Annexe 3 : Répartition des sujets selon leurs réponses aux questions 3 , 4, et 5 sur la visée de l'annonce (critique, ludique et cynique).

Faible : notes I et 2 .

Fort : notes 6 et 7 .

\begin{tabular}{|c|c|c|c|c|c|c|}
\hline & \multicolumn{2}{|c|}{ CRITIQUE } & \multicolumn{2}{|c|}{ LUDIQUE } & \multicolumn{2}{|c|}{ CYNIQUE } \\
\hline & FAIBLE & FORT & FAIBLE & FORT & FAIBLE & FORT \\
\hline TRIUMPH & $3-5-6-9-22-23$ & $8-15$ & AUCUN & $18-19-22$ & $5-15$ & $3-6-8-9-23$ \\
\hline PASSAT & $5-7-10-23$ & $\mid-8-15-17-22$ & $5-8-15-16-21-22$ & 9 & $3-7-9-15-18-25$ & $5-8-22-23$ \\
\hline AUDI & $\begin{array}{l}6-7-8-19- \\
20-22-23\end{array}$ & 17 & AUCUN & $7-9-15-19-22$ & $\begin{array}{c}4-7-9-10-11-16- \\
17-18-19-20\end{array}$ & 22 \\
\hline TRINA & $3-7-22-23$ & 22 & $3-7-8-15-23$ & 22 & $7-8-15-16-17$ & 22 \\
\hline ASPIRATEUR & $34-40-43-46$ & $47-48-49-50$ & $\begin{array}{l}32-34-40 \\
43-45-46\end{array}$ & $48-49$ & $29-40-43-46$ & $\begin{array}{l}27-33-42 \\
47-49-50\end{array}$ \\
\hline PATRIMOINE & $34-39-41-46$ & $29-33-49$ & $29-46$ & $47-49$ & $28-33-43-46-48-$ & $36-49$ \\
\hline SUZUKI & $\begin{array}{l}27-29-36 \\
39-46-49\end{array}$ & 33 & $4 \mid-46$ & $32-33-47-49$ & $43-46$ & $28-33-34-36-47-$ \\
\hline COGNAC & $40-41-44-45-46$ & $27-33-49$ & $34-44-45-46$ & $27-33-49$ & $\begin{array}{l}29-40-44 \\
46-48-49\end{array}$ & $27-33$ \\
\hline ERAM & $56-57-66$ & $63-75$ & $51-57-59-66$ & $56-62-63$ & $60-66-67-71$ & $\begin{array}{l}52-56-57 \\
63-72-74\end{array}$ \\
\hline BeEtLe & $55-6 \mid-68-72$ & $56-63$ & $55-60-66-68$ & AuCun & $55-56-66-7 \mid-75$ & $63-69$ \\
\hline PLOMBIER & $\begin{array}{c}52-53-56-57-61- \\
64-66-67-72\end{array}$ & 55 & $51-55-64-66-75$ & $56-60-62-63$ & $61-67-72$ & $52-55-56-57$ \\
\hline GAZPACHO & $\begin{array}{c}5|-6|-63-66-68- \\
7 \mid-74-75\end{array}$ & $56-74$ & $55-6|-7|-75$ & $56-74$ & $\begin{array}{c}51-61-62-63-68- \\
69-71-72-75\end{array}$ & 56 \\
\hline
\end{tabular}

